\title{
La praxis etnográfica en Educación Primaria. Experiencias desde el Museo de Artes y Costumbres Populares de Jaén
}

\author{
Ethnographic praxis in Primary Education. Experiences from the Museo \\ de Artes y Costumbres Populares de Jaén
}

\author{
María del Carmen Sánchez Miranda \\ Universidad de Jaén (España) \\ mmiranda@ujaen.es
}

Alfonso Ramírez Contreras

Universidad de Cádiz (España)

aramirezcontreras@gmail.com

José Luis Anta Félez

Universidad de Jaén (España)

jlanta@ujaen.es
Recibido 16/09/2020 Revisado 10/11/2020

Aceptado 17/11/2020 Publicado 30/11/2020

\section{Resumen:}

Desde el cuestionamiento de la relación que pueden tener niños/as con diversas procedencias del mundo dentro de un museo etnográfico, nos posicionamos entre la educación, el patrimonio y la práctica de la investigación antropológica: el marco del Museo de Artes y Costumbres Populares de Jaén se convierte en catalizador de una experiencia que, previamente comenzada en el patio del colegio, continuó en las salas del museo.

El juego había sido la referencia a utilizar y, partiendo de ella, todas las diferencias se difuminaron en el momento en que vieron en aquellas vitrinas los mismos juegos con distintos nombres de aquí y allá. Desde la decisión de llevar el museo al aula comienza la aproximación a una identidad y reconocimiento globales, configurándose el museo etnográfico como un espacio artístico integrador de culturas y generaciones.

\section{Sugerencias para citar este artículo,}

Sánchez Miranda, MªCarmen, Ramírez Contreras, Alfonso, Anta Félez, José Luis (2020).La praxis etnográfica en Educación Primaria. Experiencias desde el Museo de Artes y Costumbres Populares de Jaén Tercio Creciente (Monográfico extraordinario III), págs. 29-38, https://dx.doi.org/10.17561/rtc.extra3.5770

SÁNCHEZ MIRANDA, MCARMEN, RAMÍREZ CONTRERAS, ALFONSO, ANTA FÉLEZ, JOSÉ LUIS (2020) La praxis etnográfica en Educación Primaria. Experiencias desde el Museo de Artes y Costumbres Populares de Jaén. Tercio Creciente (Monográfico extraordinario III), noviembre 2020, pp. 29-38, https://dx.doi.org/10.17561/rtc.extra3.5770 


\section{Abstract:}

From the questioning of the relationship that children with different origins of the world may have within an Ethnographic Museum, we position ourselves between education, heritage and the practice of Anthropological research: the starting point is the Museo de Artes y Costumbres Populares de Jaen and it becomes the catalyst for an experience that, previously started in the schoolyard, continued in the Museum rooms.

The game had been the reference to be used, and based on it, all the differences were blurred at the moment they saw the same games with different names from here and there in those windows. From the decision to bring the Museum to the classroom, the approach to a global identity and recognition begins, configuring the Ethnographic Museum as an artistic space that integrates cultures and generations.

Palabras Clave: Museo Etnográfico, Educación, Cultura, Identidad, Jaén.

Key words: Museum, Education, Culture, Identity, Jaén

Sugerencias para citar este artículo,

Sánchez Miranda, $\mathrm{M}^{\mathrm{a} C a r m e n, ~ R a m i ́ r e z ~ C o n t r e r a s, ~ A l f o n s o, ~ A n t a ~ F e ́ l e z, ~ J o s e ́ ~ L u i s ~(2020) . L a ~}$ praxis etnográfica en Educación Primaria. Experiencias desde el Museo de Artes y Costumbres Populares de Jaén Tercio Creciente (Monográfico extraordinario III), págs. 29-38, https://dx.doi.org/10.17561/rtc.extra3.5770

SÁNCHEZ MIRANDA, MåCARMEN, RAMÍREZ CONTRERAS, ALFONSO, ANTA FÉLEZ, JOSÉ LUIS (2020) La praxis etnográfica en Educación Primaria. Experiencias desde el Museo de Artes y Costumbres Populares de Jaén. Tercio Creciente (Monográfico extraordinario III), noviembre 2020, pp. 29-38, https://dx.doi.org/10.17561/rtc.extra3.5770 


\section{Introducción}

Nuestra investigación etnográfica comienza con la decisión de llevar el Museo al aula; parte del contenido se entrega al alumnado para trabajar con él desde la investigación, generando curiosidad, haciendo que la visita sea deseada y no impuesta. Una decisión que proviene de nuestra propia experiencia desde la infancia y de cómo se vivenciaban aquellas visitas a lugares desconocidos de los cuales no sabíamos nada hasta que no estábamos allí.

Es por ello que no hablamos de ir al museo «porque sí» sino que esto surgirá como consecuencia de una investigación: vamos porque necesitamos una experiencia que sólo puede darse en aquel lugar. El cambio de paradigma nos despeja la duda del público cautivo y emprende un camino mucho menos desolador que derivará en un concepto que a su vez será punto de partida: el público con necesidades. Pero antes de adentrarnos en el campo práctico y en su desarrollo realizaremos una aproximación entre Etnografía y Educación (no formal), centrándonos concretamente en la relación entre ambas.

Las universidades e institutos encargados de la enseñanza e investigación de la Etnografía, cooperan generando y difundiendo la información mediante las publicaciones de sus investigadores al mismo tiempo que colaboran con las actividades escolares y de los espacios patrimoniales y museos, y en el mismo plano contribuyen organismos sin ánimo de lucro como asociaciones relacionadas con el Patrimonio, la Historia, la Arqueología y la Antropología que colaboran con actividades y proyectos de divulgación (Stone y MacKenzie, 1994; Jameson, 1997).

En este sentido, el presente trabajo recoge parte de los resultados de un proyecto de investigación que se está llevando a cabo en la Universidad de Jaén y financiado por el Instituto de Estudios Giennenses ${ }^{1}$, sobre un estudio del Museo de Artes y Costumbres Populares en la confluencia del análisis de la praxis de la etnografía con alumnado de educación primaria del Centro de Educación Infantil y Primaria Alcalá Venceslada de la ciudad de Jaén.

\footnotetext{
${ }^{1}$ Proyecto denominado «La sociedad giennense a través de la mirada del Museo de Artes y Costumbres Populares de Jaén. Un recorrido didáctico para la comprensión social de la historia», financiado por el Instituto de Estudios Giennenses, dependiente de la Diputación Provincial de Jaén, en la convocatoria de subvenciones para la investigación 2019 (Boletín Oficial de la Provincia de Jaén, Número 243, de 23 de diciembre de 2019).
} 


\section{Los museos etnográficos como espacios socioculturales y educativos: el caso del Museo de Artes y Costumbres Populares de Jaén}

Dicha investigación se deriva de una iniciativa de integración de los bienes inmateriales dentro del patrimonio cultural, como una herramienta necesaria para conocer nuestro pasado e interpretar nuestro presente a través de los museos.

Una de las primeras premisas ha sido realizar un debate sobre la funcionalidad y la razón de ser de los museos etnográficos: de su origen, de cómo se han dedicado a la exhibición, a través de diferentes objetos, a la divulgación del patrimonio inmaterial y con ello a visibilizar las raíces de las sociedades contemporáneas.

Para poder establecer una redefinición de los museos etnográficos es preciso entender el concepto de museo como un espacio de comunicación, una oportunidad de poder mostrar y enseñar al exterior una diversidad cultural de tiempos pasados y presentes. Hemos de comprender que el museo además de ser una puerta abierta a la cultura, también nos permite valorar las cuestiones de la memoria y de la identidad mediante aquellos objetos que nos han sido legados por las anteriores generaciones y que requieren de un especial interés desde un punto de vista, tanto artístico, como pedagógico, histórico, social y antropológico.

El Museo de Artes y Costumbres Populares de Jaén, ubicado en el Palacio de Villardompardo, en la capital giennense, pertenece al conglomerado de museos etnográficos, considerándose como una institución que brinda multitud de objetos, creencias y tradiciones que son las raíces de las sociedades. Sin embargo, nuestro interés abarca más allá del propio complejo museístico, estudiando la economía, la sociedad y la cultura de todos los pueblos que forman la provincia de Jaén.

Aunque el proyecto estaba planteado una década antes, esta institución fue inaugurada el 20 de diciembre del 1990, y nace con el interés de mostrar a la ciudadanía la amplia y variada cultura tradicional de la provincia, lo cual se refleja en la propia temática de la estructura del mismo:

a) Planta sótano: Salas dedicadas a la vid, olivo, cereal y agua.

b) Planta de acceso: Salas denominadas pesos y medidas y transporte.

c) Planta primera: Salas dedicadas a infancia, casa rural, sala burguesa, cerámica, cordobanes y guadamecíes y textiles.

d) Planta de cámaras: Salas de matanza, talleres y oficios artesanos y religiosidad popular. 
Parte de nuestro trabajo de análisis y revisión se apoya la propia visión que la sociedad tiene sobre los museos etnográficos. Barragán (2015) establece cómo la consideración social sobre las condiciones de vida rural, fundamentalmente agrícola y preindustrial, alejada ya en la memoria tras generaciones que no la vivieron directamente, está teñida de cierta dosis de nostalgia para un segmento de la población de mayor edad, y precisamente para ellos parecen estar diseñados esta modalidad de museos.

\section{Trazando puentes desde los museos etnográficos a las instituciones de enseñanza}

En la actualidad uno de los objetivos fundamentales en cualquier tipo de museo es captar el interés por parte de la población más joven. Yáñez (2019) afirma «actualmente en España los museos de carácter etnográfico, a nivel estatal y autonómico, se hallan inmersos en una situación de desinterés, tanto administrativamente como por parte del público»; este objetivo también aparece reflejado en las tesis de Tasky (2008), donde se promueve el vínculo entre museo-escuela.

Efectivamente, durante nuestro trabajo de investigación estamos tratando de abordar este tema, alzando el museo como una institución de enseñanza. Con ello, queremos enfatizar en la necesidad de incrementar visitas y programas educativos por parte de los centros de enseñanza infantil y primaria, estratos donde asentar bases sólidas en la formación, en la que los estudiantes no solo están realizando una actividad de ocio fuera del aula, sino que será una actividad escolar donde aprender a valorar nuestro patrimonio, fundamentalmente el patrimonio cultural inmaterial.

Asimismo, la educación para el patrimonio, según Peinado (2020) «permite a nuestro alumnado entender de dónde vienen y quienes son, lo que provoca en el alumnado una gran curiosidad y motivación por conocer más de aquello que les identifica, como: la organización social, las tendencias estéticas, las formas de vivir, sentir, vestir, comer... incluso aspectos referidos a la organización política y económica».

Como atestigua Tasky (2008) «en el vínculo con estudiantes y docentes se procura intensificar la tarea de capacitar y agilizar el diálogo que permita disfrutar del museo y 'usarlo' como herramienta educativa». Resulta de sumo interés las preguntas que nos plantea este mismo autor ¿Por qué los estudiantes en el museo? ¿Cuál es el objetivo que pretenden conseguir los museos con la participación de dichos estudiantes? Las respuestas pueden ser múltiples, pero posiblemente sea el interés del proceso de enseñanza-aprendizaje y el hecho de que el museo emerja como institución útil para la sociedad y deje de ser visto como un espacio de mera exhibición de objetos y, en nuestro caso, exóticos y anticuados para muchos de sus visitantes. 
Yáñez (2019) afirma que muchos museos etnográficos locales mantienen su actividad centrada en la exposición permanente de los fondos, con un discurso que se vuelve repetitivo, vacío de contenido, y cuya práctica museológica se torna deficiente principalmente por aspectos relacionados con la carencia de infraestructuras, presupuesto y plantilla. En este sentido, es evidente que ha surgido un cambio desde los primitivos museos etnográficos a los que nos encontramos en la actualidad, pero sigue siendo necesaria una renovación condicionada a las nuevas exigencias de las sociedades contemporáneas.

Así pues, autores como Cárdenas (2006) nos indican que han pasado más de cien años desde la apertura de los primeros museos etnográficos en Europa y así como las sociedades han cambiado, también lo han hecho estos museos, por lo que más que preguntarnos por cómo exhibir los objetos etnográficos, debemos preguntarnos por cómo mostrar las expresiones de una comunidad que en este momento vive, habla y se transforma en un devenir y eje espaciotemporal concreto.

Por tanto, estos museos tradicionalmente se han entendido como instancias dedicadas a la conservación y exhibición de la otredad, y los museos etnográficos han de adecuarse a los nuevos tiempos, buscar nuevas formas de actuación, e insertarse en el orden global, en las redes de circulación simbólica y en las narrativas de la interculturalidad (Scheiner, 2008).

\section{De disciplinas y relaciones o cómo la Educación se aproxima a la Etnografía y se contextualizan en el ámbito no formal}

Situándonos en el ejercicio de la educación no formal, apreciamos cómo ha sido desarrollada por distintas disciplinas científicas que se han visto prácticamente obligadas a ello: desde el arqueólogo que recibe a un grupo de estudiantes mientras trabaja y ha de «explicar» su tarea hasta una visita a una almazara. De cualquier manera, ésta ha sido escasamente conceptualizada y sistematizada, dejando a un lado su investigación y evaluación e impacto de modo que nos permita orientar las estrategias en relación a la transmisión del conocimiento científico de cualquiera de ellas y a su contribución en la revalorización del patrimonio ya sea arqueológico (Conforti, 2010) o de cualquier tipo.

Coombs y sus colaboradores propusieron una definición de educación no formal que ha sido ampliamente aceptada: «Cualquiera actividad educacional organizada fuera del sistema formal establecido [...] cuyo propósito es servir a clientelas identificables y objetivas de aprendizaje». 
Esta definición tiene la ventaja de establecer las características principales de la educación no formal. Consiste en actividades con las siguientes características: informales).

- Organizadas y estructuradas (de otro modo serían clasificadas como

- Diseñadas para un grupo meta identificable. aprendizaje.

- Organizadas para lograr un conjunto específico de objetivos de

- No institucionalizadas, llevadas a cabo fuera del sistema educacional establecido y orientadas a estudiantes que no están oficialmente matriculados en la escuela.

En este contexto, las prácticas etnográficas se posicionan desde un parámetro no formal sobre el que observar la intervención en patrimonio y educación.

\section{"Antes de ir al museo... que venga él": La experiencia de los alumnos del CEIP Alcalá Venceslada en el Museo de Artes y Costumbres Populares de Jaén}

Como adelantábamos en páginas anteriores, nuestra primera intención fue la de dar a conocer el Museo de Artes y Costumbres a un grupo concreto de un colegio público de la capital giennense, concretamente el CEIP Alcalá Venceslada.

Ubicado en el barrio de Belén y San Roque, este centro cuenta entre su alumnado con más de una veintena de nacionalidades que se reparten por todo el globo terráqueo: desde China a Perú, desde Marruecos a Rumanía. Las diferencias culturales son obvias y a la vez necesarias, enriquecen la convivencia y pueden ser un aspecto positivo para que el barrio crezca y prospere dentro de sus limitaciones. Pero, ¿cuál podría ser el nexo de unión entre unas y otros?

Sin entrar en los conceptos generales de cultura, ser social-cultural, ni en el de modelo cultural, sí que ahondamos en los aspectos comunes entre culturas, en concreto en uno que se reproduce a lo largo del tiempo y del espacio, que tienen en común cada una de ellas, y que se sitúa casi entre los más preciados actos de los menores de cualquier época y lugar: el juego.

Necesitábamos un nexo y lo encontramos en el mismo patio del colegio: el «pilla, pilla» también se llama «policías y ladrones» o «tú la llevas», pero finalmente se trata de que unas personas persigan a otras tan solo para luego cambiar los roles. 
Les propusimos que preguntaran en casa por juegos en sus lugares de procedencia, sus nombres y explicaciones, modos de juego y número de jugadores. Tras una sesión de introducción, todos ellos volvieron con la información y los resultados fueron emocionantes para todos: En Ecuador jugaban al mismo juego que en Mali con variaciones mínimas. Los niños y niñas se divertían jugando.

Una vez enganchados a la temática, con un objetivo común y con la predisposición a seguir aprendiendo, ahora sí, decidimos acercarnos al Museo de Artes y Costumbres de Jaén.

Allí paseamos por las distintas salas dedicadas a la infancia, volviendo a imaginar juguetes, juegos y escuchando la voz de los mayores (abuelos y abuelas presentes con el fin de «corroborar la existencia» de aquellos juguetes) que dieron buena cuenta de usos y disfrutes de los mismos ${ }^{2}$.

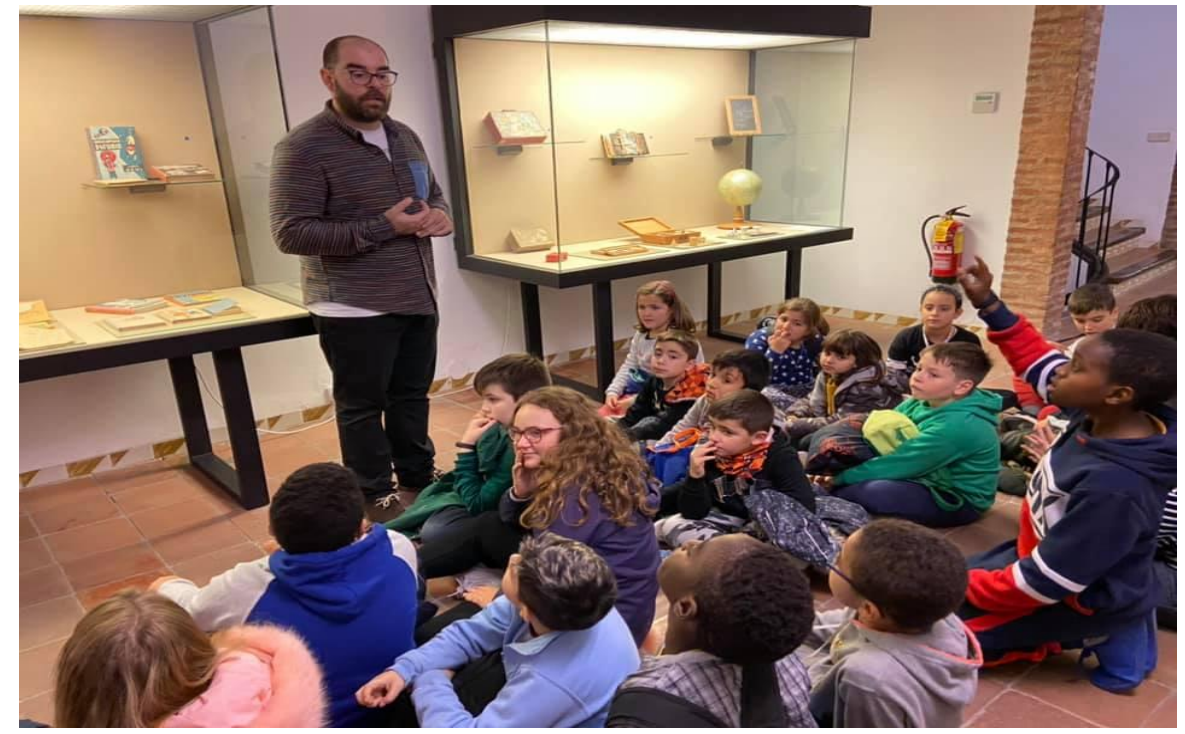

Imagen tomada del Facebook del CEIP Alcalá Venceslada (2019)

\footnotetext{
${ }^{2}$ No obstante, no quedó ahí la experiencia. Aprovechando que el edificio también alberga un museo de Arte Naíf con algunos cuadros de temática infantil (niños jugando en una plaza, por ejemplo), nos dedicamos a buscar estas obras entre las demás (con lo que era obligado el observar sin tener que obligar a observar) para discernir cuáles eran los juegos que acababan dibujados en aquellos cuadros. Y los encontramos. Y decidimos que podría ser buena idea pintar nuestros juegos. Pero eso, es otra historia.
} 


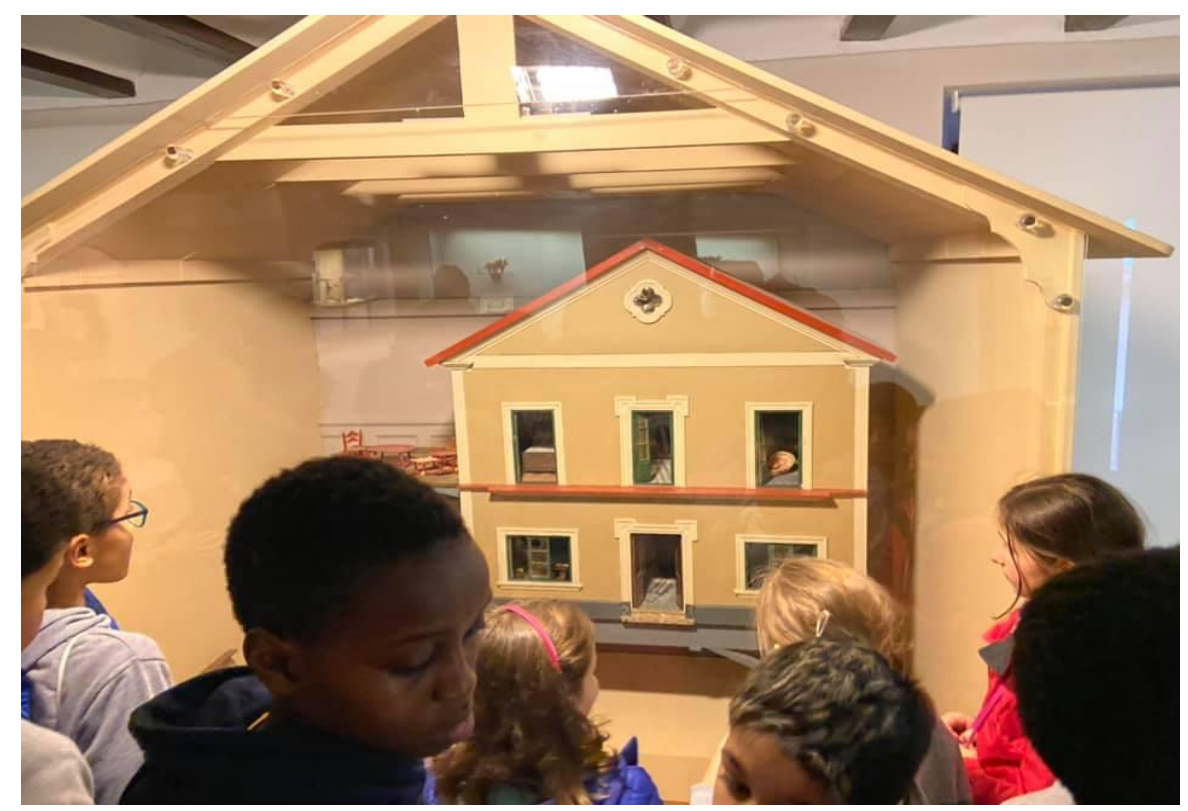

Imagen tomada del Facebook del CEIP Alcalá Venceslada (2019)

\section{Conclusiones}

Por el propio abismo generacional, los núcleos poblacionales más jóvenes otorgan escaso valor a los museos etnográficos, siendo este uno de los desafíos perseguidos, al igual que la brecha tecnológica para la transmisión de ese rico patrimonio, requiriendo una tecnología adaptada a las nuevas formas de vida de la sociedad contemporánea y que puedan ser accesibles y adaptadas a toda la población de las tendentes smart cities, donde culturalmente también es necesario avanzar.

Pero más allá de estas pinceladas sobre como la Etnografía puede entrar en algunos lugares, como disciplina educativa en niveles básicos, en el panorama que tenemos más cercano, la conexión de esta con la Educación se desarrolla sobre todo en el plano no formal y en la actualidad en el informal. Podemos ver cómo han prosperado iniciativas informales sobre todo gracias a las tecnologías de la información y la comunicación, tanto en programas divulgativos de televisión o cine, como en aplicaciones informáticas que acercan el patrimonio a la ciudadanía por muy diversos medios.

Las visitas a los museos etnográficos han de coadyuvar a la creación de una identidad plural, como herramienta para cambiar el mundo y contribuir a sociedades más comprensivas, desde el pasado, por el presente y hacia el futuro.

En definitiva, observar a los que observan, enseñarles a hacerlo mientras lo hacemos: galimatías con sentido solo apto para aquellas personas a las que les importe la aprehensión del patrimonio por parte del alumnado... sin que éste bostece y/o odie los museos para siempre. ¿Por dónde empezamos? 


\section{Referencias.}

Barragán, M. (2015). Crónica de un intento: los museos etnológicos en Andalucía. Revista andaluza de antropología, (9), 132-157. https://doi.org/10.12795/RAA.2015.i09.06

Cárdenas, B.M. (2016). Museos etnográficos. Contribuciones para una definición contemporánea (Tesis de maestría).

Conforti, M.E. (2010). Educación no formal y patrimonio arqueológico. Su articulación y conceptualización. Intersecciones en antropología 11. 103-115.

Coombs, P.H. (2006). «V». La crisis mundial de la educación. Santillana.

Jameson, J. (ed.) (1997). Presenting Archaeology to the Public. Altamira Press.

Peinado, M. (2020). Del patrimonio a la ciudadanía en Educación Infantil. Revista de Investigación e Innovación Educativa, (101), 48-57. DOI: 10.12795/IE.2020.i101.04

Scheiner, T.C. (2008). El mundo en las manos: museos y museología en la sociedad globalizada. Cuicuilco, (44), 17-36.

Stone, P., Mackenzie, R. (eds.) (1994). The Excluded Past: Archaeology in Education. Routledge.

Tasky, A. (2008). Usos del pasado, patrimonio, identidad y museos en discusión. Clío \& Asociados, 29-55. https://doi.org/10.14409/cya.v1i12.1641

Yáñez, R. (2019). Museos etnográficos de ámbito local. De la tradición al olvido. Revista andaluza de antropología, (16), 93-111. DOI: 10.12795/RAA.2019.i16.05 